\title{
Correspondence
}

Cerebrovasc Dis 2011;32:94

DOI: $10.1159 / 000327042$

\section{CT Angiography Source Images with Modern Multisection CT Scanners: Attention to Technical Principles Is Crucial}

\author{
Stephan P. Kloska $a^{\mathrm{a}, \mathrm{b}}$
}

a Department of Clinical Radiology, University of Münster Münster, and ${ }^{b}$ Department of Neuroradiology, University of Erlangen-Nuremberg, Erlangen, Germany

With interest I read the recently published article by Choi et al. [1]. The study correlated the Alberta Stroke Program Early CT Score (ASPECTS) on nonenhanced CT, conventional contrast CT (CECT) and CT angiography source images (CTA-SI) performed by modern multisection CT scanners with the pial collateral flow based on four-vessel angiography in patients with acute ischemic stroke. The authors reported that 'CECT showed more correlation with pial collateral circulation than CTA-SI' and concluded that 'ASPECTS on CECT has potential for being a stronger predictive marker of cerebral blood volume and a possible prognostic factor in acute ischemic stroke patients'. This observation is in contrast to previous reports of the blood volume basis for CTA-SI [2]. In my opinion, the reported results for CTA-SI in the article by Choi et al. [1] need further discussion, and detailed consideration of the underlying principle of CTA-SI is required.

CTA-SI, like the related techniques of perfusion-weighted CT and perfused blood volume images, are based on the principle that was first reported by Hamberg et al. [3]. With the assumption of vascular and tissue contrast steady state in CTA data, the calculation of cerebral blood volume $(\mathrm{CBV})$ can be reduced to the equation

$$
C B V=\frac{\max c(t)}{\max v(t)} \quad\left[\mathrm{ml} \cdot 100 g^{-1}\right],
$$

where $\max c(t)$ is the maximum tissue concentration and $\max v(t)$ is the maximum vascular concentration of the marker. This eliminates the need for dynamic CT data acquisition and results in qualitative information on CBV in CTA-SI. Hence, the performance of CTA data acquisition at the plateau phase of contrast injection, with vascular and tissue contrast steady, is a prerequisite. With former generations of CT scanners, dedicated injection protocols in CTA were not that crucial as the slow scan time 'au- tomatically' resulted in an appropriate bolus configuration to fulfill the algorithm of CTA-SI [2]. In contrast, the fast scan time of modern CT scanners requires very accurate bolus timing for CTA. Choi et al. [1] used a bolus tracking method and commenced the scan when enhancement in the carotid arteries reached 120 Hounsfield units, used $100 \mathrm{ml}$ of $68 \%$ nonionic contrast agent and a flow rate of $4 \mathrm{ml} / \mathrm{s}$ without saline flush. CECT was started about $80 \mathrm{~s}$ after contrast injection. Considering the fast scan time of the 16-section CT scanner used, this injection protocol results in distinct arterial contrast for CTA data. However, the mandatory assumption for CTA-SI according to the considerations by Hamberg et al. [3] is thereby violated. Hence, the size of CTA-SI lesions is overestimated. It is not surprising that Choi et al. [1] found a better correlation between CECT and dichotomized pial collateral flow compared with CTA-SI. In consequence, the results of Choi et al. [1] do not contradict the blood volume basis of CTA-SI, but point out the importance of appropriate injection protocols in conjunction with modern multisection CT scanners to fulfill the requirements for appropriate use of CTA-SI. Further comparative studies are needed to evaluate CTA-SI with appropriate injection protocols on modern multisection CT scanners for the approximation of pial collateral flow derived from four-vessel angiography.

\section{References}

1 Choi JY, Kim EJ, Hong JM, Lee SE, Lee JS, Lim YC, Kim HS: Conventional enhancement CT: a valuable tool for evaluating pial collateral flow in acute ischemic stroke. Cerebrovasc Dis 2011;31:346-352.

2 Hunter GJ, Hamberg LM, Ponzo JA, Huang-Hellinger FR, Morris PP, Rabinov J, Farkas J, Lev MH, Schaefer PW, Ogilvy CS, Schwamm L, Buonanno FS, Koroshetz WJ, Wolf GL, González RG: Assessment of cerebral perfusion and arterial anatomy in hyperacute stroke with three-dimensional functional CT: early clinical results. AJNR Am J Neuroradiol 1998;19:29-37.

- 3 Hamberg LM, Hunter GJ, Kierstead D, Lo EH, Gilberto González R, Wolf GL: Measurement of cerebral blood volume with subtraction three-dimensional functional CT. AJNR Am J Neuroradiol 1996;17: 1861-1869.

Stephan P. Kloska, MD

Department of Clinical Radiology

University of Münster, Albert-Schweitzer-Strasse 33

DE-48149 Münster (Germany)

Tel. +49 251834 7310, E-Mail kloska@ uni-muenster.de

\section{KARGER}

Fax +41613061234 E-Mail karger@karger.ch www.karger.com (c) 2011 S. Karger AG, Basel

1015-9770/11/0321-0094\$38.00/0 